\title{
DÉCLARATIONS, CONSÉCUTIVES \\ A L'ANNONCE DES RÉSULTATS DES ÉLECTIONS PRÉSIDENTIELLES EN LITUANIE ET EN FRANCE, ET CONTEXTE
}

\author{
MIROSLAV STASILO
}

Université de Vilnius, Académie militaire de Lituanie, Lituanie

\begin{abstract}
Résumé. Cet article analyse l'influence du contexte sur la langue des leaders politiques en Lituanie et en France en se basant sur l'étude de la situation politique, économique, sociale et celle des élections présidentielles entre 1993 et 2009. Nous donnons un aperçu général du contenu des allocutions politiques en nous focalisant sur le contexte des élections dans les deux pays et en nous interrogeant sur les stratégies discursives, personnelles ou politiques des intervenants analysés. Nous comparons les locuteurs en comparant leur stock lexical ainsi qu'en essayant de répondre à la question suivante: les phénomènes observés viennent-ils du contexte ou sont-ils profondément liés à la manifestation de différents éthos ? Les programmes lexicométriques Lexico 3 et Tropes nous fournissent une classification des connecteurs, opérateurs, formes de modalisation qui sont nécessaires pour notre étude pragmatique et énonciative des interventions officielles, suite à l'annonce des résultats des élections.
\end{abstract}

Mots-clés : contexte, élections présidentielles, analyse lexicométrique, vocabulaire politique, vocabulaire personnel

\section{INTRODUCTION}

Le champ politique est une 'arène' qui se donne comme telle et dans laquelle il y a des combats, des affrontements déclarés. C'est pourquoi les hommes politiques sont vulnérables au scandale (comme des hommes d'église d'ailleurs), surtout ceux qui sont au pouvoir: les scandales du financement des partis politiques de J. Chirac ou de N. Sarkozy en France, l'affaire du soutien de la campagne présidentielle de R. Paksas par l'oligarque russe N. Borisov en Lituanie. Ces scandales finissent souvent mal : J. Chirac et N. Sarkozy ont été poursuivis par le Tribunal et R. Paksas a dû démissionner. Pour décrire le fonctionnement du champ politique, on peut utiliser la métaphore théâtrale, qui permet de distinguer ce qui s'expose sur la scène et ce qui se joue en coulisses : les deux sont aussi importants. 
Le champ de nos recherches se restreint à l'analyse des textes des déclarations (abr. Déclarations) en France et des interviews (abr. Interviews), consécutives à l'annonce des résultats des élections présidentielles, en Lituanie. Notre corpus se compose de Déclarations et d'Interviews de 13 personnalités politiques (5 rançais et 8 Lituaniens) entre 1993 et 2009. Le matériau provient des sources variées : les journaux Le Monde, Le Figaro, Respublika, Lietuvos Rytas; les archives télévisuelles des chaînes de télévision en Lituanie $L R T V, L N K$ et de la BNF, l'INA en France.

Il s'agit pour nous de compter les mots des Déclarations et Interviews et de voir le contexte historique qui les entoure. Notre corpus nous fournit ainsi un matériel propice à l'analyse discursive (D. Maingueneau, P. Chareaudeau, O. Ducrot), appuyée sur le traitement lexicométrique (les programmes Lexico 3 et Tropes utilisés dans le Laboratoire du CEDITEC de l'Université Paris - Est Créteil). Nous y comparerons les locuteurs en confrontant leur stock lexical et tenterons de répondre à la question suivante : les phénomènes observés relèventils du contexte ou sont-ils profondément liés à la manifestation d'ethos différents ?

\section{CONTEXTE DES DECLARATIONS ET DES INTERVIEWS}

Les Déclarations ne sont pas en position de cadrage télévisuel (sinon directement), ni en position de dialogue face à des partenaires habilités à questionner. Les Interviews sont encadrées par la télévision et en position de dialogue avec des journalistes (qui ont le droit de poser des questions), soumis aux règles du genre d'Interviews. La situation des Déclarations et des Interviews peut paraître paradoxale : d'un côté, ces genres sont ritualisés et institutionnalisés vu l'importance de la situation des élections présidentielles dans une République 'semi-présidentielle' (Duverger, 1980) ; de l'autre, c'est un type d'expression daté, suranné, correspondant à un temps politique précis qui achève la campagne présidentielle.

Les politiques se présentent à travers leurs énonciations comme des personnes ayant gagné ou perdu au nom de leurs électeurs. L'emploi de la première personne (pronoms, adjectifs possessifs) est effectivement propre à tous les présidents élus en France et en Lituanie : 'Je mesure la difficulté de la tâche qui nous attend [...] notre bataille principale a un nom - la lutte contre le chômage' (cf. Chirac, 07-05-1998) ; 'Chacun mesure bien à l'aune de notre histoire la force de ce moment exceptionnel [...] Il y a là un espoir qui nous demande d'agrandir un espoir que je veux servir' (cf. Chirac, 05-05-2002) ; 'Je veux lancer un appel à nos partenaires européens, auxquels notre destin est profondément allié [...] Je veux lancer un appel à nos amis américains pour leur dire qu'ils peuvent compter sur notre amitié' (cf. Sarkozy, 06-05-2007) ; 'Nous marchons ensemble avec la vie. Nous la construisons ensemble, toute la Lituanie. Elle ne peut pas nous échapper, nous devons toujours la maîtriser' (trad., cf. Brazauskas, 17-01-1993) ; 'Nous avons tous le pouvoir de décider par nous-même de notre destin [...] je ferai tout pour qu'on puisse concentrer nos meilleurs forces de Lituanie' (trad., cf. Adamkus, 
05-01-1998) ; 'Nous, au moins ma famille, nous ne sommes pas venus, arrivés, soidisant, d'ailleurs. Nous sommes dans la vie politique depuis assez longtemps [...] Nous avons traversé toutes les épreuves et nous gardons toujours les mêmes: notre maison, nos amis, nos loisirs' (trad., cf. Paksas, 05-01-2003) ; 'Nous allons gagner [...] Nous ouvrons une nouvelle page de l'histoire de construction de l'Etat de Lituanie' (trad., cf. Adamkus, 24-07-2004) ; '[...] j'apprécie notre évolution dans les relations avec l'Europe occidentale, surtout au sein de l'Union européenne. Je voudrais vraiment coordonner encore plus, autrement dit, poursuivre le progrès acquis grâce au Président actuel, qu'on observe en ce moment' (trad., cf. Grybauskaite, 17-05-2009).

Il faut cependant distinguer l'emploi de toutes ces formes, par ex., 'je' et 'nous', en fonction de la différence stratégique des énoncés analysés dans un contexte historique et électoral varié. Ainsi, l'emploi massif du pronom de la première personne de Chirac (17 fois en 1998 et 19 en 2002) ou de Sarkozy (44 ! fois) n'a pas la même valeur que celui de Le Pen ( 9 fois) ou de Royal (20 fois). Les premiers deux locuteurs appartenaient au groupe de candidats gagnants avec un statut plus élevé, qui leur permettait d'associer ou de lier plus facilement leurs caractéristiques personnelles au destin et à l'image de tout le pays. Alors que les candidats vaincus avaient recours au pronom de la première personne ayant eu envie de souligner leur distinction individuelle, qui n'a pas permis de gagner des élections données mais qui était un garant de victoires à venir.

Or, le même groupe de locuteurs emploie le 'je' et le 'nous' différemment selon des moments historiques différents. Ainsi Jacques Chirac, leader du parti du Rassemblement pour la République (RPR), avait-il remporté les élections présidentielles de 1995 avec plus de 52\% des voix quand les Guignols, sur Canal Plus, se moquaient à travers le slogan 'Mangez des pommes' contre Lionel Jospin, croqué en Oui-Oui, et Edouard Balladur, considéré comme 'traître'. Chirac a mené sa 3ème campagne présidentielle sous le symbole du pommier contre la 'facture sociale' et pour la 'responsabilité de l'Etat français' avec l'élection de Bille Clinton aux Etats-Unis en 1992 et le retour de la droite au pouvoir en France grâce aux élections législatives en 1993 ainsi que la cohabitation 'de velours' entre Fr. Mitterrand et Edouard Balladur, la nomination de Nicolas Sarkozy en tant que Ministre du Budget. L'emploi du pronom de la première personne par Chirac est donc assez personnel en 1995. Alors que son utilisation de la même forme est plus collective car le moment historique n'était pas le même : les thèmes principaux de la campagne présidentielle de 2002 étaient le 'chômage' et la 'délinquance', la situation de la cohabitation (cette fois-ci entre J. Chirac et L. Jospin) a commencé à être considérée plutôt comme une anomalie et non comme une pacification du pouvoir politique en France, la campagne électorale était l'une des moins médiatisées à cause du résultat préliminaire 'connu' d'avance et de la baisse de l'intérêt des électeurs à la vie politique. Mais grâce à une faible participation de l'électorat français, c'est Jean-Marie Le Pen qui avait gagné contre Jospin (16,86\% contre $16,18 \%$ ) au premier tour des élections. Un tel résultat était inattendu et avait choqué non seulement toute la France mais aussi le monde entier. Le 'je' ou 
'nous' de Chirac n'était pas donc qu'une association à tout le peuple français mais aussi une opposition commune à la représentation personnelle de Le Pen.

On a la même situation dans le cas des locuteurs lituaniens qui emploient aussi souvent les pronoms ou adjectifs de la première personne en fonction de la différence énonciative d'un moment et d'un locuteur choisi. Par exemple, Brazauskas emploi le 'je' ou 'nous' moins souvent que les autres candidats élus : Adamkus, Paksas et Grybauskaite. La situation économique et politique du pays était difficile en 1993 : la monnaie russe 'rouble' n'était plus la devise nationale du pays, la Russie poursuivait sa politique de la négligence de la Lituanie sur l'arène internationale, la privatisation continuait à gagner du terrain dans tous les domaines, l'institution présidentielle en Lituanie a été instaurée en 1992 avec la proclamation de l'indépendance et par l'adoption de la Constitution, Algirdas Mykolas Brazauskas a été élu Président du Parlement et il a pris aussi les fonctions du Président par intérim jusquaux élections présidentielles du 14 février 1993 qu'il a gagnées avec un score record - 60,03\% de votes pour sa candidature. Dans un tel contexte, l'emploi de 'je' et de 'nous' ( 16 fois en total) est moins fréquent que le substantif 'les gens' (zmonés en lit.), qu'on retrouve quatorze fois dans l'interview de Brazauskas en 1993.

Adamkus a pu utiliser le 'je' et le 'nous' plus fréquemment - onze fois en 1998 et vingt fois en 2004. En 1998, il y a eu une forte opposition idéologique entre le champ de gauche avec le Président sortant en tête et les partis de droite sans un leader qui aurait le même poids qu'A. M. Brazauskas. V. Adamkus n'avait 'lancé' son expérience présidentielle en Lituanie qu'en 1993, quand il avait dirigé la campagne électorale de Stasys Lozoraitis. Ensuite en 1996 il a rassemblé les forces modérées pour gagner les élections législatives de 1996 et plus tard, en 1998 - les élections présidentielles. Valdas Adamkus a été élu Président de la République de Lituanie le 26 février 1998. Son gouvernement était plus actif et indépendant que celui d'A. M. Brazauskas, même si le taux de ses électeurs était moins élevé $50,4 \%$ (37,1\% de tous les électeurs) en dépassant son rival seulement $0,7 \%$. Le motclef de la campagne présidentielle et de sa gouvernance était 'la modernisation rapide du pays' (en 2003, on lui a attribué le titre d'Ambassadeur de bonne volonté de l'Unesco pour avoir créé en Lituanie une société bien informatisée) et 'la représentation de tout le peuple'.

Le programme de traitement lexicométrique 'Lexico 3' nous permet de rédiger la liste des mots-clés $(\mathrm{MC})$ plus complète en incluant deux parties du corpus - français et lituanien (cf. Tableaux 1,2):

\section{Tableau 1 Mots-clés des Déclarations}

\begin{tabular}{|l|l|}
\hline Formes & Fréquence \\
\hline De, à, pour, dans, en, au, avec, par, aux, entre, contre, & $\begin{array}{l}199,77,47,34,33,18,15,11,9,9, \\
6,6(=464)\end{array}$ \\
sur (prép.) & $146,75,71,33,63(=388)$ \\
\hline La, les, le, l' (art. défini) & \\
\hline
\end{tabular}




\begin{tabular}{|l|l|}
\hline Formes & Fréquence \\
\hline Je (j'), ma, mon, mes; sa, son, ses; nous, notre, nos; & $\begin{array}{l}109(76+33), 15,7,18 ; 7,6,8 ; 28, \\
18,5 ; 22,4,3 ; 26,9(=268)\end{array}$ \\
\hline vous, votre, vos; leur, leurs (pron.) & $61,59(=120)$ \\
\hline Qui, que & $100,15,4(=119)$ \\
\hline Et, mais, où (conj.) & $41,32(=73)$ \\
\hline France, français (Français, Française) & $5,34,2,9 ; 3,12,8(=71)$ \\
\hline $\begin{array}{l}\text { Suis, est, sommes, sont; serai, sera, seront (verbe } \\
\text { 'être') }\end{array}$ & $36,19(=55)$ \\
\hline Un, une (art. indéfini) & $29,1,1,1(=32)$ \\
\hline Veux, voulons, voulez, veulent (verbe 'vouloir') & 16 \\
\hline République & 13 \\
\hline Compatriotes & $11,1,1(=13)$ \\
\hline Chers, cher, chères & $5,3,2,2$ \\
\hline Aujourd'hui, avenir, maintenant, demain & 9 \\
\hline Monde & $3,4(=7)$ \\
\hline Europe, européen (européenne) & 7 \\
\hline Etat & 7 \\
\hline Appel & \\
\hline
\end{tabular}

\section{Tableau 2 Mots-clés des Interviews}

\begin{tabular}{|c|c|}
\hline Formes & Fréquence \\
\hline Ir, kad, bet, ar, o, nes, vis (conjonctions) & $287,179,39,35,18,16,8(=582)$ \\
\hline $\begin{array}{l}\text { As, tu, jis, ji, savo, musu, man, mano, jie, mes, ju, jus, } \\
\text { juos, jums, juo, jusu (pronoms et adjectifs personnels) }\end{array}$ & $\begin{array}{l}150,8,12,11,30,54,31,38,29 \\
21,16,7,4,2,1,1(=415)\end{array}$ \\
\hline $\begin{array}{l}\text { Taip, labai, kaip, tikrai, ne, cia, tik, dar, daug, tiek, } \\
\text { daugiau ( } \underline{\text { adverbes }})\end{array}$ & $\begin{array}{l}61,55,53,42,38,30,29,29,18,16, \\
14(=385)\end{array}$ \\
\hline $\begin{array}{l}\text { Yra, buvo, bus, butu, buti, esu, buvau, busiu, esam, } \\
\text { esame, esas, budamas, buta, buve, buna (verbe 'être') }\end{array}$ & $\begin{array}{l}94,51,34,26,11,10,6,3,2,2,1,1, \\
1,1,1(=244)\end{array}$ \\
\hline Tai, ta, kas, kurie ('cela') & $104,61,29,25,21(=240)$ \\
\hline $\mathrm{Su}, \mathrm{i}$, is, apie, uz, pries (prépositions) & $52,40,35,25,19,18(=189)$ \\
\hline Reiskia, manau, 'sakyti', turi (verbes 'penser', 'dire') & $30,30,33,21(=114)$ \\
\hline $\begin{array}{l}\text { Zmones, zmoniu, zmonems, zmogus, zmogaus, } \\
\text { zmogu, zmogumi, zmogui, zmogiska (substantif } \\
\text { 'gens') }\end{array}$ & $33,29,11,5,5,2,2,1,1(=89)$ \\
\hline $\begin{array}{l}\text { Lietuvos, Lietuva, Lietuvoje, Lietuvoj, Lietuvai, } \\
\text { lietuviai, lietuviams, lietuviskas, lietuviska (subst. } \\
\text { 'Lituanie') }\end{array}$ & $54,12,9,6,4,1,1,1,1(=89)$ \\
\hline $\begin{array}{l}\text { Visiems, visi, visus, visa, visu, viskas, visai, visas, } \\
\text { viso, visoms, visos, visame, visiem, visoj ('tout, toute, } \\
\text { tous, toutes') }\end{array}$ & $\begin{array}{l}17,16,10,8,8,4,3,3,2,2,2,1,1 \\
1(=78)\end{array}$ \\
\hline
\end{tabular}




\begin{tabular}{|l|l|}
\hline $\begin{array}{l}\text { Prezidento, prezidentas, prezidentu, prezidenta, } \\
\text { prezidentura, prezidenturoje, prezidentais, } \\
\text { prezidente, prezidentui, prezidenturai, prezidentui, } \\
\text { prezidentus (subst. 'président') }\end{array}$ & $\begin{array}{l}12,10,3,2,2,2,1,1,1,1,1, \\
1(=37)\end{array}$ \\
\hline $\begin{array}{l}\text { Politikoje, politika, politikos, politiku, politine, } \\
\text { politiniu, politikai, politikas, politinemis, } \\
\text { politiniame, politikais, politines, politiniai, politinio, } \\
\text { politinis, politinius (subst. 'politique') }\end{array}$ & $\begin{array}{l}7,5,5,3,3,3,2,2,2,2,1,1,1,1,1, \\
1(=37)\end{array}$ \\
\hline $\begin{array}{l}\text { Valstybes, valstybe, valstybiu, valsybei, valstybeje, } \\
\text { valstybems, valstybine, valstybines, valsybingumu, } \\
\text { valstybiniame ('Etat') }\end{array}$ & $14,2,2,1,1,1,1,1,1,1(=25)$ \\
\hline $\begin{array}{l}\text { Dabar, dabartinis, daznai, ateities, ateityje, pabaiga, } \\
\text { siandien ('maintenant') }\end{array}$ & $12,2,2,2,2,2,2(=24)$ \\
\hline Europos, Europai, europinems, Europoj ('Europe') & $7,1,1,1(=10)$ \\
\hline
\end{tabular}

Les fréquences des MC montrent le suremploi de prépositions dans le cas des orateurs français (464) et de conjonctions - dans le cas lituanien (582). Cela est dû à la différence des langues : le français est une langue analytique alors que le lituanien est synthétique, endomorphique. Or les politiques lituaniens et français ont un recours également excessif à l'emploi des pronoms (415 et 268), étant donné la coïncidence des fonctions de cataphores et anaphores dans ces deux langues: participer à la cohérence du texte en évitant les répétitions lexicales. Cela démontre aussi une présence fréquente des pronoms ou adjectifs possessifs de la première personne, employés souvent pour légitimer son statut élevé ainsi que présenter son appartenance à un groupe commun (N.B. : d'après Le Bart, le discours politique implique quatre présupposés dont la légitimation de l'autorité et l'addition des citoyens à une communauté (Le Bart, 1998 : 70)).

\section{VOCABULAIRE POLITIQUE}

La plupart des gens pensent que la politique, c'est toujours la même chose (Poškienè, 2008). Grâce aux programmes Lexico 3 et Tropes, on distingue ces univers sémantiques (US) : France, monde, mouvement, force, français, citoyen, désir, nation, compatriotes, parti, changement, fierté, avenir, droits de l'homme, combat, histoire, méditerranée, peuple, pays, patrie, métropole, respect (cf. Tableaux 1, 2). Ces mots appartiennent au champ sémantique des valeurs universelles qu'on rencontre souvent dans un style fleuri, abstrait, soi-disant franc et sincère, qui peut s'apparenter à la 'la langue de bois' (Chosson, 2007).

Les données statistiques de Benoît Deschayes, accessibles sur Internet (En ligne 1), avaient démontré les vocables des politiques français les plus fréquents à cette époque. On pourra les comparer avec nos résultats. On y rencontre souvent les mêmes formes: pronoms de la première personne 'je' ou 'nous', pronom de la deuxième personne 'vous' avec les verbes 'être' et 'vouloir' 
qui témoignent de la volonté énonciative de légitimer son statut, les substantifs 'France', 'République', 'monde' et les adjectifs 'français', 'européen' qui parlent de la politique intérieure et extérieure (N.B. : les affaires intérieures intéressent plus nos énonciateurs). Mais ily a aussi des différences. Les orateurs analysés emploient également des vocables qu'on ne retrouve pas forcément chez Deschayes, par exemple, les substantifs 'compatriotes' (13) et 'Etat' (7), l'adjectif 'cher' (13), inévitables si on veut remercier les électeurs de leur soutien ou en parlant au nom de l'Etat (surtout les présidents élus).

A la seule lecture de la liste de MC ci-dessus, on saisit aisément la volonté des politiques des deux pays d'utiliser des vocables compréhensibles, abstraits et généraux : France, République, compatriotes, Lietuva (Lituanie), Prezidentas (Président), Valstybe (Etat), pasaulis (monde), Europa (Europe). On peut retrouver également chez les orateurs lituaniens quelques mots étrangers : 'interesavosi' (chez Brazauskas, ce verbe vient du verbe russe 'interesovatsia', s'intéresser), 'iseliminuosim' (chez Brazauskas, ce verbe est aussi assez souvent utilisé en russe et il provient du verbe français éliminer) 'Yankee, go home', 'actually' (chez Adamkus), 'egzetpulai' (chez Grybauskaite, ce nom vient d'une expression anglaise 'exit poll' et signifie des résultats préliminaires), 'de facto, de jure' (chez Grybauskaite, cette expression est d'origine latine et c'est un terme juridique). Les mots étrangers sont absents dans les Déclarations puisque ce genre discursif est plus rigide et codifié que les Interviews.

Les orateurs français parlent davantage des symboles historiques, par exemple, des valeurs républicaines : terre de liberté, de fraternité, d'égalité des chances, terre de solidarité ; valeurs de la République ; grands idéaux, fidèle à sa vocation universelle et humaniste ; une grande, à une vieille, à une belle nation, la France ; la République les devoirs de respect et d'égalité qu'elle a envers eux. Ils mentionnent peu de personnalités historiques ou politiques. Par contre, les orateurs lituaniens font davantage référence à leurs prédécesseurs politiques qui symbolisent l'Etat lituanien, et aux actualités du moment, surtout dans le cas des candidats battus. En tous cas, les présidents élus et les candidats vaincus des deux pays comprennent l'importance de ce rituel républicain pour la légitimation de leur statut et pour mettre en valeur la situation de communication particulière ainsi que leur ethos. On verra ci-dessus des différences et ressemblances des MC personnels dans les deux pays.

\section{VOCABULAIRE PERSONNEL}

Les MC sont liés au sens des énoncés qui ne reflètent pas que des événements historiques mais sont également des commentaires du 'dire' (Ducrot, 1984) qui caractérisent des 'sujets parlants' (ibidem). Les données lexicométriques distinguent les énoncés de Sarkozy en France et d'Adamkus en Lituanie. On retrouve dans la Déclaration du premier énonciateur les six premiers substantifs de sa liste des MC traditionnels : 'France' (24 fois), 'Français' (10), 'Monde' (5), 'République' (3), 'Président' (2), 'Travail' (1). Le septième nom de sa liste 
traditionnelle, 'Etat', est absent, remplacé par 'France' ou 'République' dans la Déclaration : 'Maintenant, c'est à mon tour de rendre à la France ce que la France m’a donné' ; '[...] que chacun puisse y trouver sa place dans notre République, que chacun s'y sente reconnu', etc. (cf. Sarkozy, 06-05-2007). Ceci marque la volonté de Sarkozy de se différencier de Chirac qui s'affiche très étatiste.

On ne retrouve pas non plus le nom 'Françaises' dans sa Déclaration alors que ce mot est dans sa liste chez Deschayes (cf. ci-dessus). Cela peut être justifié par son appartenance à l'UMP, parti de droite, qui ne traite pas spontanément les questions sociales, telles la parité entre les sexes ou encore l'égalité des chances. Pour compenser ce manque, l'orateur parle des 'femmes' (3 fois dans la Déclaration). Nicolas Sarkozy ne veut pas se montrer machiste et affiche qu'il respecte les femmes. La présence assez nombreuse des femmes dans le gouvernement (et dans sa vie - trois mariages) peut aussi justifier cette remarque. Une autre illustration en est le remerciement, dès le début de la Déclaration, à sa concurrente Ségolène Royal : 'Et ma pensée va à Madame Royal. Je veux lui dire que j'ai du respect pour elle et pour ses idées, dans lesquelles tant de Français se sont reconnus' (cf. Sarkozy, 06-05-2007). Sarkozy prononce deux fois 'Madame Royal' dans sa Déclaration. Il présente ainsi son respect qui est assez démonstratif et un peu 'vieux jeu' grâce à l'appel 'Madame'. Le langage de la Déclaration de Sarkozy est assez varié, élevé et soutenu mais moins emphatique et glorieux que celui de Chirac. Sarkozy parle plus clairement en refusant un style allégorique et les constructions syntaxiques qui perdent l'auditeur et le citoyen. Ce qui est peutêtre le résultat de ses rédacteurs des textes, dont $\mathrm{H}$. Guaino.

Quant aux énoncés d'Adamkus, ses énoncés se distinguent des autres grâce aussi au suremploi des formes : il dit le plus souvent le pronom 'as' (je, moi 39 fois) et le substantif 'Lietuva' (Lituanie - 27). C'est aussi lui qui tisse le plus fréquemment des liens énonciatifs via la conjonction de subordination 'kad' (que - 37). C'est l'Interview d'Adamkus en 2003 qui se distingue fortement des autres suite à l'emploi massif des formes du pronom de la première personne je ('as') et nous ('mes') ainsi que des noms : Lietuva (Lituanie) et zmones (gens), du pronom visi (tous); des verbes : sakyti (dire), reiskia (signifie) et manau (je pense). Cette distinction est liée au contexte des élections tendues et imprévisibles jusqu'au dernier moment de l'annonce des résultats. Concernant ses énoncés de 1998 et de 2004, il n'y pas de traits particuliers sauf peut-être l'usage du substantif 'Lietuva', des formes du pronom tous ('visi') et des verbes avoir ('tureti') avec 'reiskia' (signifie) que l'énonciateur ne néglige pas.

L'Interview d'Adamkus se distingue grâce aussi à son statut vaincu qui permet et oblige les politiques de parler plus de la situation actuelle, par exemple, critiquer la société, le déroulement des élections, les adversaires, etc. Voici les MC des candidats battus en Lituanie: 1) Lozoraitis: 'Brazauskas', 'zmones' (gens), 'jis' (lui), 'Amerika' (Etats-Unis), 'jie' (eux), 'Bobelis', 'pinigai' (argent), 'Lietuva', 'ambasada' (ambassade), 'opozicija' (opposition), 'Adamkus', 'Maskva', 'kaimas' (campagne) ; 2) Paulauskas: 'pergale' (victoire), 'rinkimai' 
(élections), 'zmones' (gens), 'visi' (tous), 'abejones' (doutes), 'rezultatai' (résultats), 'Lietuva', 'Prezidentas' (Président), 'balsai' (voix), 'idejos' (idées), 'Valstybe' (Etat), 'Adamkus' , 'Brazauskas', 'balsavimas' (vote) ; 3) Adamkus (en 2003) : 'as' (moi), 'Lietuva', 'zmones' (gens), 'mes' (nous), 'visi' (tous), 'rinkimai' (élections), 'sprendimas' (décision), 'Valstybe' (Etat), 'Prezidentas' (Président), 'problemos' (problèmes), 'kampanija' (campagne électorale), 'visuomene' (société), 'darbas' (travail), 'politika' (politique), 'atsakomybe' (responsabilité), 'pareiga' (devoir), 'vyriausybe' (gouvernement) ; 4) Prunksiene: 'as' (moi), 'jie' (eux), 'jis' (lui), 'kompetencija' (compétence), 'Panevezys', 'Europa', 'Maskva', 'seimas' (parlement), 'visi' (tous); 5) Butkevicius : 'as' (moi), 'visuomene' (société), 'seimas' (parlement), 'vyriausybe' (gouvernement), 'darbas' (travail), 'salis' (pays), 'mintys' (idées), 'Prezidentas' (Président), 'kandidatas' (candidat), 'komitetas' (comité), 'pasitikejimas' (confiance), 'specialistas' (professionnel), 'sprendimas' (décision), 'debatai' (débats), 'problema' (problème), 'Grybauskaite', 'Lietuva', 'konsolidacija' (consolidation), 'Europa'.

Les thèmes (et les MC par conséquence) des candidats vaincus lituaniens se ressemblent puisqu'ils consacrent beaucoup d'attention au déroulement et à l'entourage géopolitique de la campagne des élections présidentielles, aux personnalités politiques : on y trouve les pronoms de la troisième personne ; les noms propres ; les substantifs comme 'parlement, gouvernement, Etat', etc. (cf. cidessus). Tous les candidats vaincus utilisent le substantif généralisant 'zmones' (gens) mais ils parlent moins des thèmes globaux comme 'responsabilités, vie, Etat, devoirs, politique', etc., en préférant des thèmes problématiques comme 'argent, résultats du vote, travail'. Cela est dû au genre des Interviews et à leur statut vaincu qui obligent d'aborder des sujets plus concrets.

Le statut gagnant permet de toucher aux politiques lituaniens des thèmes globaux: 1) Brazauskas: 'zmones' (gens), 'as' (moi), 'mes' (nous), 'Lietuva', Prezidentas' (Président), 'partija' (parti politique), 'Lozoraitis', 'rinkimai' (élections), 'Valstybe' (Etat), 'vyriausybe' (gouvernement), 'visi' (tous), 'jie' (eux), 'Konstitucija' (Constitution), 'ekonomika' (économie), 'gyvenimas' (vie); 2) Adamkus (en 1998) : 'Lietuva', 'as' (moi), 'zmones' (gens), 'visi' (tous), 'darbas' (travail), 'vyriausybe' (gouvernement), 'gyvenimas' (vie), 'politika' (politique), 'Amerika' (Etats-Unis), 'Paulauskas', 'mes' (nous), 'atsakomybe' (responsabilité), 'santykiai' (relations), 'NATO' (OTAN) ; 3) Paksas : 'zmones' (gens), 'Lietuva', 'uzsienis' (étranger), 'salis' (pays), 'politika' (politique), 'Adamkus', 'visi' (tous), 'mes' (nous), 'valstybe' (Etat), 'visuomene' (société), 'kompetencija' (compétence), 'pareigos' (devoirs), 'Europa', 'Sajunga' (Union), 'NATO' (OTAN), 'seimas' (parlement) ; 4) Adamkus (en 2004): 'as' (moi), 'Lietuva', 'zmones' (gens), 'mes' (nous), 'jis' (lui), 'valstybe' (Etat), 'Prezidentas' (Président), 'gyvenimas' (vie); 5) Grybauskaite: 'Lietuva', 'as' (moi), 'zmones' (gens), 'ministrai' (ministres), 'jie' (eux), 'darbas' (travail), 'premjeras' (Premier ministre), 'Europa', 'mes' (nous), 'salis' (pays), 'jus' (vous), 'nuosmukis' (crise), 'politika' (politique), 'seimas' (parlement), 'atsakomybe' (responsabilité), 'partija' (parti politique). 
Si l'on compare ces thèmes avec ceux des présidents élus en France, on remarque que les politiques français préfèrent plus les thèmes liés à la République, à l'humanisme, à l'éternité. Ils emploient fréquemment des substantifs comme 'République', 'monde', 'liberté', 'liberté', 'démocratie', etc. Pourquoi les politiciens français tant aiment-ils la langue de bois, trop abstraite et menteuse ? Lorsque les orateurs politiques parlent de grands thèmes, par exemple, du racisme, de la tolérance, du pacifisme, de l'égalité entre les femmes et les hommes, il est difficile de choisir les mots exacts, d'émettre des idées suffisamment nuancées pour ne blesser personne. C'est pourquoi le langage devient plus abstrait en parlant des valeurs démocratiques qui servent à cacher parfois une certaine impuissance des politiques (Charaudeau, 2005).

\section{CONCLUSIONS}

Malgré le contexte historique et national différent, on peut conclure que les politiciens des deux pays ont en commun deux axes sémantiques. Une position est liée à la personnalité et l'autre - au parti ou aux intérêts politiques représentés. La position personnelle parle de l'éthique, des questions culturelles. Alors que les principes politiques sont ancrés dans la réflexion sur la Constitution, la démocratie, l'Etat, la nation, le pays. Les ethos des politiques s'opposent et cela ne dépend ni du statut ni du parti politique. C'est le contexte du moment qui décide du caractère de l'opposition. Les présidents élus s'opposent ainsi aux candidats vaincus : Chirac à Jospin et Le Pen, Sarkozy à Royal, Brazauskas à Lozoraitis, Adamkus à Paulauskas, Paksas et Prunskiene, Grybauskaite à Butkevicius.

Nos orateurs parlent des valeurs démocratiques et se présentent comme les garants de la liberté. Les orateurs sont influencés par l'opinion publique et le contenu discursif tourne autour de la réaction aux résultats du vote. Il n'y a pas d'effets sociaux très importants parce que deux genres appartiennent au rituel des élections. Cependant la particularité générique des interviews permet aux énoncés lituaniens de faire valoir le contexte mieux que le genre de déclarations consécutives à l’annonce des résultats présidentielles.

\section{BIBLIOGRAPHIE}

Charaudeau, P. (2005) Le discours politique. Les masques du pouvoir. Paris : Vuibert.

Chosson, M. (2007) Parlez-vous la langue de bois ? Petit traité de manipulation à l'usage des innocents. Paris : Points.

Ducrot, O. (1984) Le dire et le dit. Paris : Minuit.

Duverger, M. (1980) A new political system model : Semi-presidential government. European Journal of Political Research, 8 (2): 165-187.

Le Bart, C. (1998) Que sais-je ? Le discours politique. Paris : PUF.

Poškienè, A. (2008) Politika kaip komunikacinis žaidimas. Vilnius: Vilniaus Universiteto leidykla. 


\title{
SITES INTERNET
}

[En ligne 1] Accessible sur https://www.linternaute.com/actualite/politique/1162635-lesmots-des-politiques/ [consulté le 15 novembre 2019].

\section{SOURCES ANALYSEES}

Le Figaro, 1995-2007

Le Monde, 1995-2007

Libération, 1995-2007

Lietuvos aidas, 1993-2009

Lietuvos Rytas, 1993-2009

Respublika, 1993-2009

\section{DECLARATIONS CONSECUTIVE TO THE ANNOUNCEMENT OF THE RESULTS OF THE PRESIDENTIAL ELECTIONS IN LITHUANIA AND FRANCE, AND THE CONTEXT}

\begin{abstract}
This article analyzes the influence of context on the language of political leaders in Lithuania and France based on the study of the political, economic, social situation and that of the presidential elections between 1993 and 2009. We give a general overview of the biggest historical changes as well as the context of elections in both countries by questioning the personal and political discursive strategies of the speakers analyzed. We compare speakers by comparing their lexical stock by attempting to answer the following question: do the observed phenomena come from the context or are they deeply related to the manifestation of different ethos? The lexicometric programs Lexico 3 and Tropes provide us with a classification of the connectors, operators and forms of modalization which are necessary for our pragmatic and enunciative study of the official interventions following the announcement of the results of the elections.
\end{abstract}

Key words: context, presidential elections, lexicometric processing, political vocabulary, personal vocabulary

Miroslav Stasilo (Dr. en Sciences du langage) travaille actuellement à l'université de Vilnius comme assistant scientifique et à l'académie militaire de Lituanie comme enseignant-lecteur. Ses centres d'intérêt professionnel sont l'analyse du discours et l'enseignement du français langue étrangère (FLE). Courriel : miroslav.stasilo@lka.lt 